ÉGYPTE monde arabe

\section{Égypte/Monde arabe}

$11 \mid 2014$

Ville et révolution en Égypte

\title{
La planification controversée du Grand Caire avant/après 2011
}

Randa A. Mahmoud et Ahmed S. Abd Elrahman

\section{(2) OpenEdition}

\section{Journals}

Édition électronique

URL : https://journals.openedition.org/ema/3305

DOI : 10.4000/ema.3305

ISSN : 2090-7273

\section{Éditeur}

CEDEJ - Centre d'études et de documentation économiques juridiques et sociales

\section{Édition imprimée}

Date de publication : 6 avril 2014

Pagination : 177-201

ISBN : 978-2-905838-84-1

ISSN : $1110-5097$

\section{Référence électronique}

Randa A. Mahmoud et Ahmed S. Abd Elrahman, "La planification controversée du Grand Caire avant/ après 2011 », Égypte/Monde arabe [En ligne], 11 | 2014, mis en ligne le 11 avril 2014, consulté le 07 juillet 2022. URL : http://journals.openedition.org/ema/3305 ; DOI : https://doi.org/10.4000/ema.3305 
Randa A. Mahmoud \& Ahmed S. Abd Elrahman

\section{LA PLANIFICATION CONTROVERSEE DU GRAND CAIRE AVANT/APRES 2011}

\section{RÉSUMÉ}

La révolution de 2011 a suscité chez les Egyptiens un sentiment nouveau de patriotisme. Parallèlement, la société civile se montre de plus en plus décidée à reprendre son avenir en main. Quelques ONG commencent ainsi à prendre part au débat sur le développement urbain du Caire et de l'Egypte. Des nouvelles stratégies ont été formulées ou reconsidérées: " Development Corridors», "Egypt 712 », " Egypt Vision : 2030 ». Simultanément, les responsables politiques de I'aménagement du territoire réalisent l'inapplicabilité des plans stratégiques directeurs jusqu'à présent conçus pour Le Caire. En réaction au surinvestissement de la capitale par l'ancien régime, Le Caire n'est aujourd'hui plus le point focal du nouveau plan stratégique directeur " Egypt 2052 » conçu par le GOPP, organe chargé de la planification du territoire égyptien. Presque du jour au lendemain, le GOPP s'est convaincu que s'éloigner du Caire serait la solution à tous les problèmes d'aménagement que rencontre l'Egypte. Très loin du "Grand Caire $2050 »$ (précédent grand plan stratégique directeur) et de tous les remèdes proposés afin de résoudre les problèmes du Grand Caire, nous sommes aujourd'hui face à un document de planification dans lequel la capitale égyptienne est rarement mentionnée.

Cette recherche met, tout d'abord, en lumière les paradoxes et les enjeux des nouvelles stratégies proposées, soit par la société civile ou par le GOPP, avant de s'interroger sur leur efficacité, et leur validité dans le contexte égyptien.

MOTS CLÉS :

GOPP, Cairo 2050, Egypt 2052, corridors de développement, Egypt 712. 


\section{ABSTRACT}

The revolution of 2011 aroused among the Egyptians a new sense of patriotism. Meanwhile, the civil society shows more determined to take charge of its future. Some NGOs begin to take part in the debate on "Cairo and Egypt's urban development". New strategies have been formulated or reconsidered: "Development Corridors», "Egypt 712», «Egypt Vision: 2030». Simultaneously, decision makers of strategic planning realize the inapplicability of strategic plans so far designed for Cairo. In response to overinvestment in the capital by the former regime, Cairo today is no longer the focal point of the new strategic plan «Egypt 2052», proposed by the GOPP (responsible organization for planning the Egyptian territory). Almost suddenly, the GOPP is convinced that moving away from Cairo would solve all development problems facing Egypt. Very far from the «Cairo 2050» (previous general strategic plan) and all proposed to solve the problems of Greater Cairo remedies, we are now faced with a strategic planning document in which the Egyptian capital is rarely mentioned.

This research highlights, first, the paradoxes and challenges of new strategies proposed by either the civil society or the GOPP before questioning their effectiveness and validity in the Egyptian context.

\section{KEYWORDS:}

GOPP, Cairo 2050, Egypt 2052, Development Corridors, Egypt 712. 


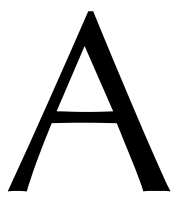

gglomération polycentrique dès plus son jeune âge, la capitale égyptienne a connu différentes phases d'aménagement spécifiques afin d'absorber une croissance démographique spectaculaire. Elle est ainsi passée d'un noyau de 5 hectares en 624, appelé Fustat, à une ville de 12000 hectares en 1952 et, enfin, à un espace mégalopolitain de 52400 hectares et 17 millions d'habitants en 2010'.

Le Grand Caire cumule aujourd'hui les indices de vulnérabilité urbaine tandis que sa polarisation à l'échelle de l'Egypte reste intacte, du fait de l'hyper-concentration des opportunités d'emploi.

Depuis les années 1960, en raison des carences des pouvoirs publics en matière de logement et de services urbains, la dichotomie formel/ informel ne cesse de s'accentuer. Le recensement de 2006 a montré que le secteur informel a cru de 2,57 \% par an depuis 1996, tandis que la "ville formelle » ne s'est accrue que de 0,4 \% par an sur la même période. Perçues comme un signal d'alarme par bon nombre d'acteurs, ces évolutions vont donner lieu à un certain nombre de réflexions stratégiques et de propositions d'encadrement de la croissance urbaine du Grand Caire qui se multiplient dans les années 2000.

Depuis la révolution de 2011, les débats sur la planification du Grand Caire sont, à l'instar d'autres types de débat public, très controversés. Le projet "Cairo 2050 », porté par le gouvernement de Moubarak et abandonné à sa chute, envisageait du faire du Caire une "ville mondiale, globale, verte et connectée ", en en renforçant la centralité à toutes les échelles. En conséquence, le General Office for Physical Planning (GOPP), chargé de l'aménagement du territoire égyptien, a mis sur la table un nouveau schéma directeur nommé «Egypt 2052 ». Dans ce dernier, le Caire n'est pas au premier plan, l'objectif étant de réussir la décentralisation promue par les nouveaux pouvoirs.

Dans le cours de l'année 2011, des documents de planification alternatifs ont été élaborés par des ONG : "Egypt 712 » et "Egypt Vision : 2030 ", par exemple. De même, le plan d'aménagement imaginé par Farouk El-Baz dans les années 1990 et intitulé «Les Corridors du Développement » est remis au gout du jour, après avoir été longtemps boycotté sous le régime de Moubarak.

1. Barthel P.-A., "Relire le Grand Caire au miroir de la densité », Confluences Méditerranée, $\mathrm{n}^{\circ} 75,2010$. 
Entre ces différentes visions, de nombreuses questions peuvent être formulées : sur quelles bases réelles travailleront les aménageurs du Caire de demain? Le schéma directeur «Egypt 2052 » conçu en quelques mois par un gouvernement de transition est-il réellement applicable ou reste-t-il, à l'instar de son prédécesseur "Cairo 2050 », bien plus une vision d'ensemble qu'un plan d'aménagement du territoire ? La solution peut-elle réellement venir de la société civile, actuellement en pleine restructuration et également traversée par des divergences de vue?

Le premier gouvernement civil représenté par les Frères musulmans avait redonné espoir aux milieux de l'urbanisme et à la société civile impliquée dans le débat urbain. Néanmoins, aucun changement, ni aucune distance vis-à-vis des politiques urbaines de l'ancien régime, n'ont réellement été ressentis par l'ensemble de ces acteurs au cours de l'année d'exercice de pouvoir par les Frères musulmans. Aucune stratégie digne de ce nom n'a par ailleurs été mise en œuvre depuis la révolution.

La destitution de Mohamed Morsi en juillet 2013 et l'installation $d^{\prime}$ une nouvelle administration technocratique n'ont pas engendré, à ce jour, de nouvelles directions politiques en matière de développement urbain. Au contraire, cette instabilité politique tend à faire passer au second plan les questions d'aménagement urbain pourtant cruciales aujourd'hui dans une agglomération comme Le Caire qu'il devient urgent de repenser dans sa totalité.

\section{LE CAIRE EN 2013 : CAPITALE DU DYSFONCTIONNEMENT URBAIN?}

Le Grand Caire est aujourd'hui I'une des métropoles les plus densément peuplées au monde avec 17 millions d'habitants en 2010. En revanche, la quantité d'espaces publics et d'espaces verts y est l'une des plus faibles au monde. Ces derniers ont pratiquement disparu sous l'effet de la construction d'infrastructures routières (ponts, autoponts, ronds-points, échangeurs, etc.) au cours de ces dernières décennies, qui a également eu pour conséquence l'augmentation constante du nombre de véhicules individuels dans la ville (Elnaggar et alii, 2006).

Le Grand Caire abrite une part importante de la population égyptienne. En 2006, la population cairote représentait $23 \%$ de la population du Pays (contre $18,6 \%$ en 1996) et $43 \%$ de la population urbaine (contre $30 \%$ en 1996). La population du Caire a doublé et sa superficie a triplé en 25 ans (El Kadi, 2011). Après la seconde Guerre Mondiale, Le Caire a été soumise à un fort mouvement migratoire provenant des villes et villages de Haute-Egypte. Les migrants étaient attirés par le développement 
économique de la capitale, renforcé avec la politique d'industrialisation lancée par Nasser. Si l'exode rural a longtemps constitué le principal facteur de l'expansion urbaine du Caire, des études récentes montrent que ce n'est plus réellement le cas, la croissance naturelle ayant pris le dessus (Kipper, 2009). Les migrants ruraux ne constitueraient plus aujourd'hui que $5 \%$ de la croissance de la capitale Egyptienne.

Par ailleurs, si le Grand Caire gagne chaque année de nouveaux habitants, la ville centre en perd. La part de la population résidant à I'intérieur des limites du gouvernorat du Caire est ainsi passée de 13,8 $\%$ en 1976 à 10,7 \% en 2006, en raison d'un mouvement centrifuge ayant transporté les populations vers les périphéries urbaines des autres gouvernorats : Giza et Qalyubiya (MOHUUD, JICA, GOPP, 2008).

Le Grand Caire occupe une position stratégique dans le nord de I'Egypte, mise en valeur par les réseaux de transports interrégionaux tels que la route d'Alexandrie et celle d'Ismaïlia, connectées entre elles par la rocade (ring road) du Caire. Des extensions urbaines et industrielles se sont développées le long de ces grands axes, aggravant la congestion de la capitale. Il n'existe aucune structure macroadministrative autonome chargée de gérer l'ensemble des problèmes précités à l'échelle du grand Caire, alors même que le GOPP établit I'existence du grand Caire comme échelle pertinente pour l'action territoriale (Sims, 2003).

En dépit des appels incessants à la décentralisation (voir l'article de $M$. Nada dans ce dossier), les administrations et les sièges gouvernementaux sont encore très majoritairement concentrés dans la capitale. Le Grand Caire symbolise également la centralisation absolue en ce qui concerne les activités tertiaires de haut niveau: médias, maisons d'édition, en plus des plus grands hôpitaux et universités (UNHabitat, 2011). C'est est aussi le principal médiateur culturel, agent et diffuseur - dans le monde arabe et le Moyen-Orient - de la production musicale, littéraire, vidéo et cinématographique. De même, la plupart des industries égyptiennes sont situées au Caire où se concentrent également $43 \%$ des emplois publics, $40 \%$ des emplois privés et 49 $\%$ des emplois industriels. En outre, la création de zones industrielles dans les villes nouvelles telles que Six Octobre et Dix de Ramadan fait du Grand Caire le point focal de la plupart des activités de fabrication moderne. L'économie du Grand Caire domine aujourd'hui près de la moitié du PIB de l'Egypte.

Parallèlement à cette économie formelle hyper-centralisée, le grand Caire a également vu se développer une immense économie informelle qui comprend des milliers de micro-entreprises. Cette économie semble avoir connu un boom depuis la Révolution de 2011. On observe en effet dans la plupart des quartiers de la capitale, et même au-delà, une augmentation des constructions illégales (Sims, 2003). 
D'anciens bâtiments ont été démolis et remplacés par des tours édifiées sans permis de construire. De même, la surface des terres agricoles est en constante diminution au nord de l'agglomération, en direction du delta du Nil (Sims, 2012). A ce problème de l'informel (plus de 60 $\%$ de l'aire urbaine du Grand Caire aujourd'hui) s'ajoutent, au titre des vulnérabilités urbaines, I'hyperdensité de la ville, I'augmentation des prix du foncier, la congestion de la circulation, la dégradation de l'environnement, etc. (Tarbush, 2012 ; Kipper, 2009).

\section{EVOLUTION DE LA PLANIFICATION DU GRAND CAIRE DEPUIS 1949}

Dans les années 1940, les affaires urbaines sont contrôlées par les ministères centraux. II n'existe alors aucune forme de planification urbaine stratégique. Seule la "loi de lotissement» de 1940 encadre le développement urbain par le biais de normes européennes très rigoureuses concernant la disposition des îlots, des bâtiments et des espaces publics (Sims, 2003). A cette époque, aucun projet de logement n'est financé par le gouvernement afin de faire face à la croissance démographique dont on mesure déjà les premiers effets sur la pression foncière (UN-Habitat, 2011).

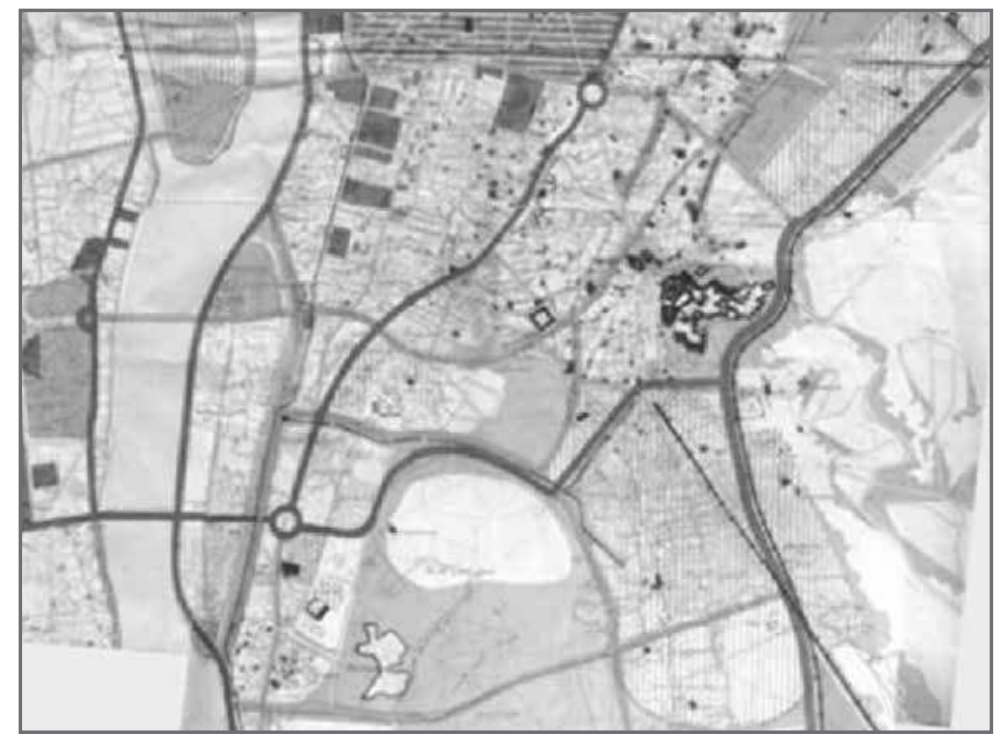

Figure 1 : Le Schéma Directeur de la Région du Grand Caire de 1956 (GOPP, 2010). 
En 1949, la Municipalité du Caire est créée. Elle deviendra I'Administration du Logement et des Services Publics du Gouvernorat du Caire en 1960. Le premier schéma directeur du Caire (Figure 1) a été élaboré en 1956 dans le but d'aider la ville à absorber sa croissance interne endémique. Ce schéma prévoit la nécessité d'orienter la croissance à l'est et à l'ouest, dans le désert en somme. Consécutivement, un programme gouvernemental de logements sociaux est lancé et doit concerner 4,5 millions d'habitants (Piffero, 2009). Cet objectif n'a jamais été atteint. En 1958, le gouvernement de Nasser a quant à lui lancé le programme d'urbanisation ambitieux de «Nasr City» sur la poche désertique située entre Le Caire et Héliopolis. Il donne lieu à la création d'une puis de plusieurs puissantes sociétés foncières mandatées par les Ministères du Logement et de la Défense (propriétaire des terrains) qui développeront ensuite les quartiers Héliopolis, Maadi et Ain Shams (Sims, 2003).

Si le schéma directeur de 1956, tout comme sa réédition en 1970, préconisent déjà la décongestion de la capitale et la redistribution de la population sur des terres non agricoles, I'urbanisation du désert, à travers le modèle des villes nouvelles, $\mathrm{n}^{\prime}$ a pris sa forme concrète qu'au milieu des années 1970. La régulation de la croissance urbaine devient alors la responsabilité du GOPP. En 1975, le gouvernement cède de nouvelles parcelles de terrain et offre des facilités financières à différents organismes de promotion immobilière qui se multiplient.

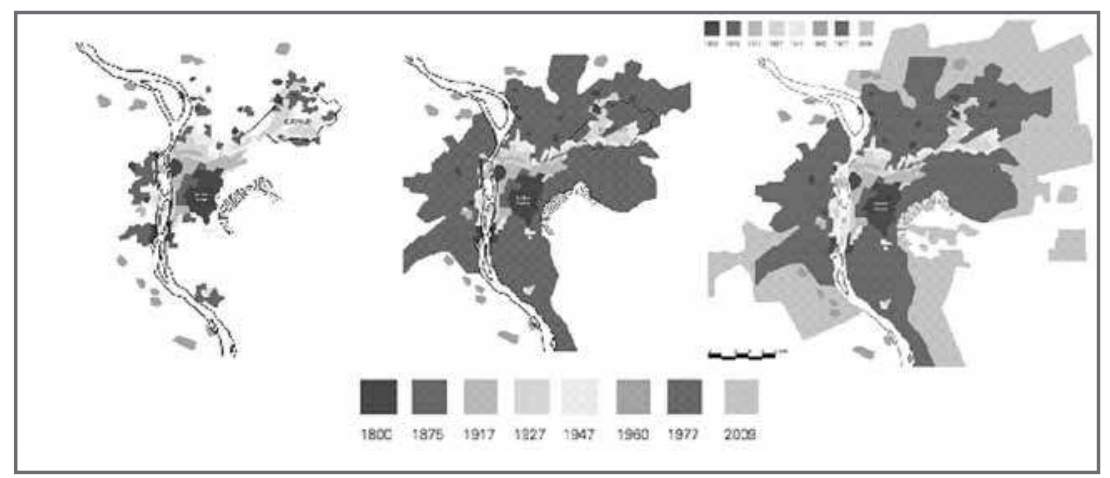

Figure 2 : L'Evolution urbaine de l'agglomération de Grand Caire, Source : Fait par les Etudiants de KU Leuven pendant un Atelier sur Héliopolis à I'Université Ain Shams, 2010, Modifiée par les chercheurs. 
En 1979, le gouvernement lance une politique de grands projets : celle des villes nouvelles, encadrée par l'Autorité des Nouvelles Communautés Urbaines. Depuis cette date, des ressources nationales importantes ont été allouées à ces « New Towns » (World Bank, 2008). Entre 1998 et 2002, 22 \% du budget national du Ministry of Housing, Utilities and Urban Development (MHUUD), ont été consacrés aux New Urban Communities Authority (NUCA), tandis que $2 \%$ seulement de la population urbaine réside dans les villes nouvelles au cours de cette période (UN-Habitat, 2011).

Le schéma directeur de 1970, mais aussi celui de 1983, abordait déjà la question du défi démographique de la capitale tout en cherchant à limiter l'expansion informelle sur les terres agricoles. L'aménagement des villes nouvelles et l'amélioration des conditions de vie dans les zones mal desservies étaient alors les priorités de cette nouvelle politique planificatrice. Les solutions proposées s'achevaient par la nécessité de réduire les distances de déplacement et de limiter la circulation routière. "Mais, plus de trente ans après, le bilan de cette politique est très modeste, car ces villes nouvelles ont du mal à se remplir tandis que l'urbanisation des terres agricoles ne cesse de progresser »(El Kadi, 2011).

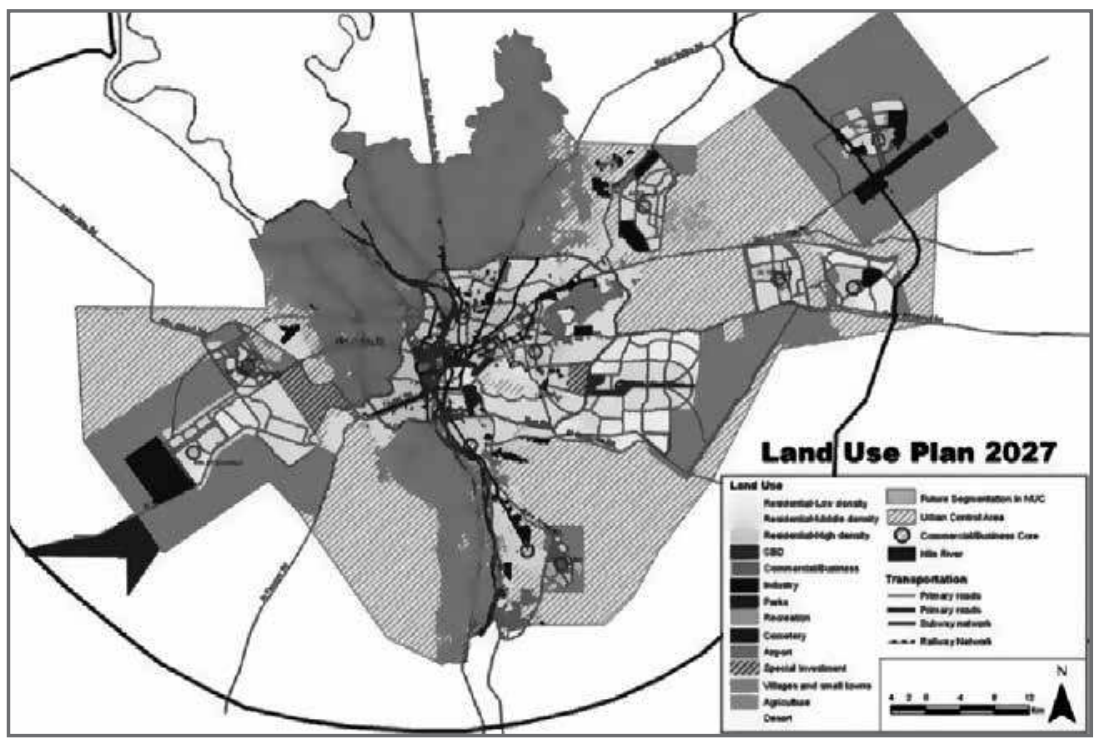

Figure 3 : Plan d'aménagement du territoire du Grand Caire 2027 (Source : GOPP, 2008). 
En 1993, le gouvernement créée le "National Fund for Urban Upgrading " afin de réhabiliter les zones d'urbanisation informelle qui progressent de façon spectaculaire. Le Fond a cependant concentré ses efforts sur quelques grands projets d'infrastructures sans proposer de réelles solutions pour les tissus urbains auto-construits. La croissance de l'urbanisation informelle dans le Grand Caire a malgré tout ralenti dans une certaine mesure, non pas grâce à la politique de réhabilitation mais au fléchissement de la croissance démographique annuelle, qui passe de 2,8 \% entre 1976 et 1986 à 1,8 \% entre 1986 et 1996 (Kipper, 2009).

\begin{tabular}{|c|c|}
\hline 1940 & $\begin{array}{l}\text { Aucun mécanisme de planification } \\
\text { Loi de lotissement }\left(n^{\circ} 52\right) \text { pour réguler le } \\
\text { développement urbain }\end{array}$ \\
\hline 1949 & Mise en place de la municipalité du Caire \\
\hline 1956 & Elaboration du premier schéma directeur \\
\hline 1958 & Lancement du plan de Nasr City \\
\hline 1960 & $\begin{array}{l}\text { Remplacement de la municipalité par } \\
\text { l'Administration du Logement et des } \\
\text { Services Publics du Gouvernorat du Caire }\end{array}$ \\
\hline 1966 & $\begin{array}{l}\text { Création de quatre sociétés de logement: } \\
\text { Nasr City, Héliopolis, Maadi et Ain Shams }\end{array}$ \\
\hline 1970 & Elaboration du deuxième schéma directeur \\
\hline 1973 & Création du GOPP \\
\hline 1975 & $\begin{array}{l}\text { Ouverture de nouvelles voies de } \\
\text { développement en fournissant des facilités } \\
\text { financières au secteur immobilier (politique } \\
\text { d'Infitah de Sadate) }\end{array}$ \\
\hline 1979 & $\begin{array}{l}\text { Décret d'application de la loi sur les } \\
\text { Nouvelles Communautés Urbaines } \\
\end{array}$ \\
\hline 1983 & Elaboration du troisième schéma directeur \\
\hline 1993 & $\begin{array}{l}\text { Mise en place du "National Fund for Urban } \\
\text { Upgrading" }\end{array}$ \\
\hline 2008 & $\begin{array}{l}\text { Elaboration des schémas directeur Cairo } \\
\text { 2027, puis Cairo 2050, et création du } \\
\text { Informal Settlements Development Fund }\end{array}$ \\
\hline
\end{tabular}

Figure 4: Ligne du temps pour l'évolution de la planification urbaine du Grand Caire. 
Le développement des villes nouvelles s'est donc très vite imposé comme la seule et unique réponse apportée par les pouvoirs publics à la croissance démographique. On dénombre alors huit principaux pôles du développement établis à la périphérie du Caire pour absorber des millions de ménages à faibles et moyens revenus. Le plan de l'Autorité pour les Nouvelles Communautés Urbaines prévoyait un réseau très sophistiqué de transport en commun, contenant un système de métro connecté aux lignes ferroviaires régionales, des couloirs de bus et des ponts. Cependant, aucun des ces plans n'a été mis en œuvre, et la distance qui sépare le centre-ville de ces villes nouvelles demeure un frein à leur développement (UN-Habitat, 2011).

Jusqu'au début du nouveau millénaire, le développement du Grand Caire n'est pas encadré par une stratégie d'aménagement du territoire à proprement parler et les politiques urbaines semblent se limiter d'une part à une réhabilitation au compte goutte des zones informelles et d'autre part au développement des villes nouvelles. Il faut attendre l'année 2008 pour que le GOPP rende public un nouveau plan stratégique d'aménagement du territoire, lequel se projette sur l'année cible de 2027. Cette stratégie (Figure 4) identifie 16 grandes catégories d'occupation du sol associées à une forme urbaine spécifique. Cette stratégie sectorielle et fonctionnaliste comprend par ailleurs 52 projets et programmes classés en trois phases de délai de mise en œuvre : court terme (au cours de cinq prochaines années), moyen terme (au cours de dix prochaines années) et long terme (au cours de vingt prochaines années) (MOHUUD, JICA, GOPP, 2008).

\section{UN PROJET DE PLANIFICATION URBAINE MODERNISTE}

\section{Cairo 2050}

En 2008, quelque mois seulement après la publication du document Cairo 2027, le gouvernement égyptien dévoile Cairo 2050, un plan plus ambitieux visant à transformer le Caire en " ville verte, globale et connectée ", au même titre que "Tokyo, Paris ou Londres ». Ce projet reconsidère l'ensemble de la planification urbaine du Caire développée depuis les années 1970 (Barthel, Monqid, 2011). Rêve urbain de l'ancien régime, Cairo 2050 est un projet évalué à 3,5 million de dollars. II a été directement commandé par Moubarak estimant que Cairo 2027 n'allait pas assez loin dans la prospective. Ce projet a également reçu I'appui technique de nombreuses organisations dont le Programme du Développement des Nations Unies, le Programme des Etablissements Humains de Nations Unies, la Banque Mondiale, coopération étatique allemande (GIZ) et l'Agence Japonaise de Coopération Internationale (Tarbush, 2012). 


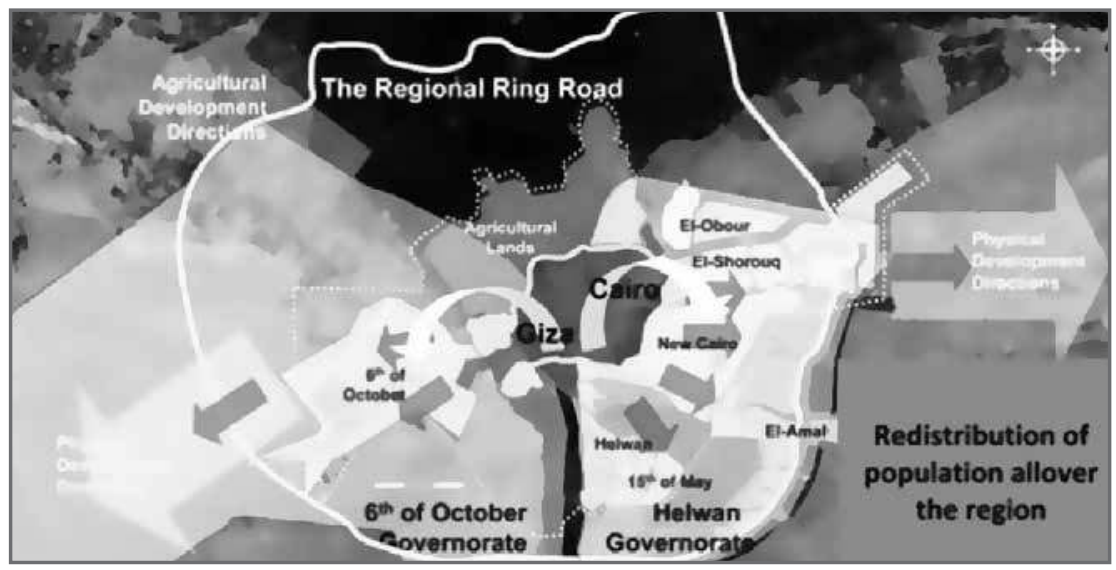

Figure 5 : Redistribution de la population à l'échelle du Grand Caire (Cairo 2050) (GOPP, 2010).

Le gouvernement affirme qu'une approche participative a été mise en place pour élaborer ce projet, à travers des consultations qui duraient 9 mois environ (GOPP, 2010). Ainsi, plus de 1800 planificateurs, hauts responsables et intellectuels auraient participé au processus, lors d'ateliers, de symposiums et de conférences. Certains médias se sont également enthousiasmés pour ce projet qui affirme prendre en compte tous les aspects du développement durable: le volet environnemental (lutte contre la pollution, création de parcs et jardins), le volet social (formation, éducation à l'environnement), le volet économique (préservation des terres agricoles) (Barthel, Monqid, 2011). Les prévisions démographiques de ce projet estimant à 30 millions la population du Grand Caire à I'horizon 2050, la conquête du désert demeure la solution, visant à épargner les terres encore cultivées mais aussi à reloger en périphérie les habitants des zones $d^{\prime}$ urbanisation informelle (Figure 5). Le centre-ville devrait ainsi accueillir de nouveaux éléments de la modernité urbaine comme des parcs d'affaires et de loisirs, des hôtels de luxe, des centres touristiques, des tours de bureaux et de larges boulevards. La compétitivité économique de la ville serait ainsi à son plus haut niveau, incarnée par un certain nombre de mégaprojets reliés entre eux par un système de transport public intégré (Madbouly, 2010). Les autorités ont en effet justifié le lancement de Cairo 2050 afin d'aider Le Caire à conserver son rôle de «première capitale du Moyen-Orient ».

De nombreuses voix se sont élevées, notamment après la révolution de 2011, pour critiquer le plan Cairo 2050, en ce qu'il soulève un sentiment d'inégalité socio-économique à l'échelle du pays. Focalisé en effet sur le Caire en tant que système mégalopolitain centralisé, 
les pouvoirs publics, à travers cet unique document stratégique, n'abordent pas le développement des villes des autres gouvernorats. Plusieurs critiques sont également basées sur le fait que ce plan fut principalement pensé par et pour les catégories sociales supérieures de la population et dans le cadre d'un schéma de collusion entre intérêts gouvernementaux et non publics. Quoi qu'il en soit, le projet, dévoilé par de nombreux sites internet, semble avoir irrité la population, à un moment de gronde sociale de grande ampleur.

\section{RÉVOLUTION DE 2011 : UN TOURNANT POUR LA PLANIFICATION URBAINE?}

Peu après la révolution de 2011, les autorités responsables de la planification urbaine du Caire changent complètement leurs visions du développement de la capitale égyptienne. La décentralisation semble être devenue un nouveau leitmotiv pour les cadres dirigeants du GOPP, y compris pour ceux déjà en place sous Moubarak au sein de cette même institution. Ainsi, en contradiction avec tout ce qui a été conçu sous l'ancien régime, une nouvelle stratégie d'ensemble appelé Egypt 2052 a été imaginée par la GOPP, afin de rééquilibrer le territoire national (développement des pôles urbains secondaires et limitation de l'expansion du Caire) et de poursuivre l'urbanisation des zones désertiques.

Highlighting the Egyptian Location in the Region and Worldwide.

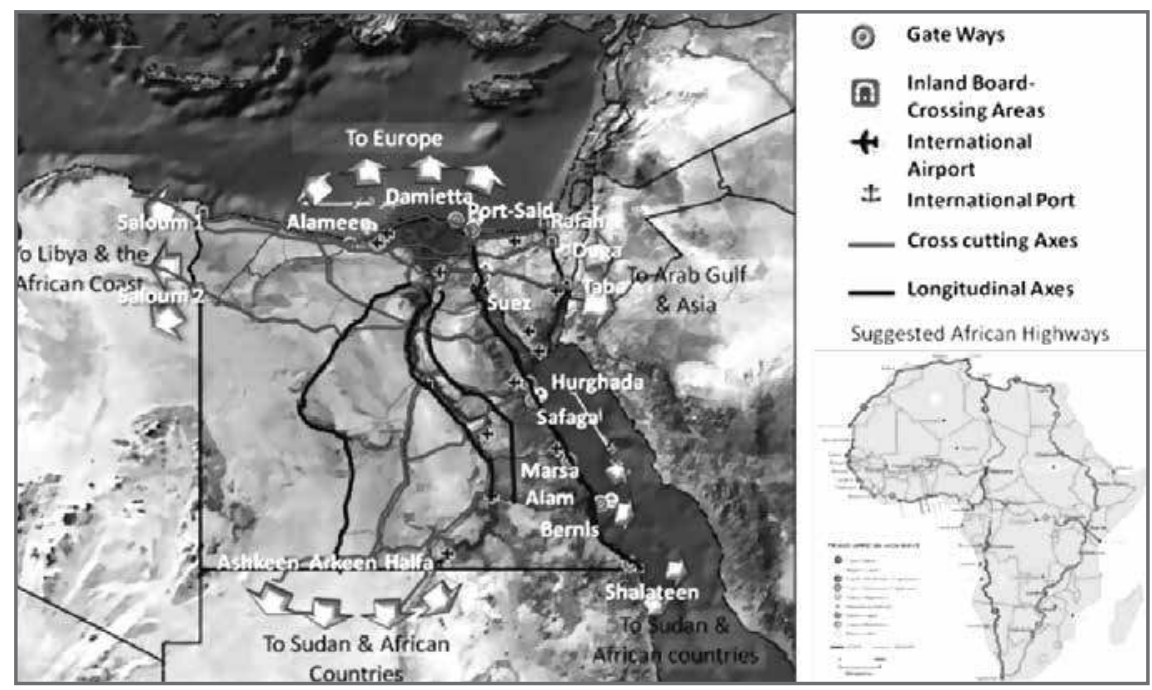

Figure 6 : Document de promotion du territoire égyptien, (GOPP, 2011).

2. 1 Feddan $=4200 \mathrm{~m}^{2}$. 


\section{Egypt 2052}

A travers ce projet du GOPP, les auteurs imaginent une Egypte " développée et compétitive ", une "nation pionnière, possédant de vastes potentialités économiques, une équité sociale, une démocratie locale forte et une meilleure qualité de vie pour toute la population» (Figure 6).

La stratégie Egypt 2052 se base sur l'état de la répartition de la population sur le territoire pour développer son argumentation. Le document rappelle donc que les 80 millions d'égyptiens n'occupent que $6 \%$ de la superficie totale du territoire $\left(1\right.$ million de $\left.\mathrm{Km}^{2}\right)$; ils sont essentiellement regroupés dans le Delta et la Vallée du Nil qui constituent $74 \%$ de la surface urbanisée du pays.

Les planificateurs font par ailleurs l'inventaire des principaux obstacles au développement urbain en Egypte : le défi de ressources humaines $(22 \%$ de pauvreté, $29,6 \%$ d'analphabétisme et $10 \%$ de chômage); le défi économique (faible disponibilité des ressources telles que l'eau, le gaz et le pétrole); le défi territorial (extrême concentration de la population; disparition des zones agricoles sous l'effet de l'urbanisation non réglementaire).

Le plan Egypt 2052 souhaite ainsi concentrer ses efforts sur l'efficacité économique, grâce à l'utilisation maximale des ressources ; sur l'équité sociale en luttant contre la pauvreté et en rééquilibrant la répartition de la population; et enfin, sur la lutte contre les menaces naturelles, surtout la sécheresse et le développement des énergies renouvelables.

Plusieurs études d'impact auraient déjà été réalisées dans le cadre de la préparation de cette stratégie, sur le tourisme, le commerce, les activités agricoles et le développement industriel. Il en résulte une série d'indicateurs présentés dans le tableau suivant, sensés révéler, à court, moyen et long terme, la faisabilité du plan. 


\begin{tabular}{|l|l|l|l|l|}
\hline \multicolumn{1}{|c|}{ Indicateur } & $\begin{array}{c}\text { Situation } \\
\text { existante }\end{array}$ & $\begin{array}{c}\text { L'Égypte } \\
\text { dans 5 ans }\end{array}$ & $\begin{array}{c}\text { L'Égypte } \\
\text { dans 15 ans }\end{array}$ & $\begin{array}{c}\text { L'Égypte } \\
\text { dans 40 } \\
\text { ans }\end{array}$ \\
\hline $\begin{array}{l}\text { La croissance } \\
\text { démographique }\end{array}$ & $2,04 \%$ & $1,85 \%$ & $1,6 \%$ & $1,3 \%$ \\
\hline Le taux d'analphabétisme & $29,6 \%$ & $17 \%$ & $6 \%$ & $6 \%$ \\
\hline $\begin{array}{l}\text { Taux de pauvreté de la } \\
\text { famille }\end{array}$ & $22 \%$ & $15 \%$ & $10 \%$ & $2 \%$ \\
\hline Taux de chômage & $10 \%$ & $8 \%$ & $7 \%$ & $6 \%$ \\
\hline $\begin{array}{l}\text { Numéro de bidonvilles des } \\
\text { zones dangereuses }\end{array}$ & 295 & 0 & 0 & 0 \\
\hline $\begin{array}{l}\text { Terres cultivées (Million } \\
\text { Feddan' }\end{array}$ & 8,8 & 9,5 & 11,7 & 14,7 \\
\hline Pourcentage d'eau dessalée & $0,24 \%$ & $0,3 \%$ & $5 \%$ & $20 \%$ \\
\hline $\begin{array}{l}\text { Pourcentage de nouvelles } \\
\text { ressources énergétiques }\end{array}$ & $10 \%$ & $12 \%$ & $15 \%$ & $25 \%$ \\
\hline $\begin{array}{l}\text { Longueur des réseaux } \\
\text { routiers nationaux }\end{array}$ & $16215 \mathrm{Km}$ & & $20000 \mathrm{Km}$ & \\
\hline $\begin{array}{l}\text { Longueur du réseau } \\
\text { ferroviaire à grande vitesse }\end{array}$ & $6410 \mathrm{Km}$ & & $3110 \mathrm{Km}$ & \\
\hline
\end{tabular}

Source : « Egypt 2052 », GOPP, 2011.

Le GOPP invite par ailleurs le gouvernement à adopter au préalable un programme de développement national conditionnant la faisabilité de Egypt 2052, afin d'améliorer la qualité de vie de la population au cours des 5 prochaines années. Les priorités sont les suivantes: utilisation de la recherche scientifique en matière de dessalement et d'utilisation de l'énergie solaire ; lutte contre l'analphabétisme ; mise à niveau de l'enseignement technique ; éradication des zones d'insécurité urbaine, etc.

Si la stratégie Egypt 2052 aborde les problèmes de façon globale et systémique, sa principale faiblesse réside dans son approche du territoire national. Elle se focalise surtout sur la sortie de l'étroite vallée du Nil en envisageant de développer de grands axes tels que la Côte Nord, I'ouest du Sinaï et le Canal du Suez. Elle prévoit également de faire de l'axe Le Caire/Aïn Sukhna/Suez une vaste zone logistique, commerciale et de services (Figure 7). Néanmoins, le rayonnement du Grand Caire sur le territoire n'est que très peu mentionné dans ce projet, en dépit de tous les problèmes urbains et sociaux qui y existent. En outre, certaines propositions de ce plan peuvent sembler irréalistes, notamment celle 
qui consiste à éradiquer " 295 zones urbaines dangereuses » dans les cinq années à venir, sans mentionner concrètement les moyens pour y parvenir ni de quelle catégorie d'espace il s'agit.

Developing the Eastern Desert and connecting Cairo and Suez canal Regions Developing Cairo-Suez-Soukhna Axis as Logistic, Commercial \& Service Areas

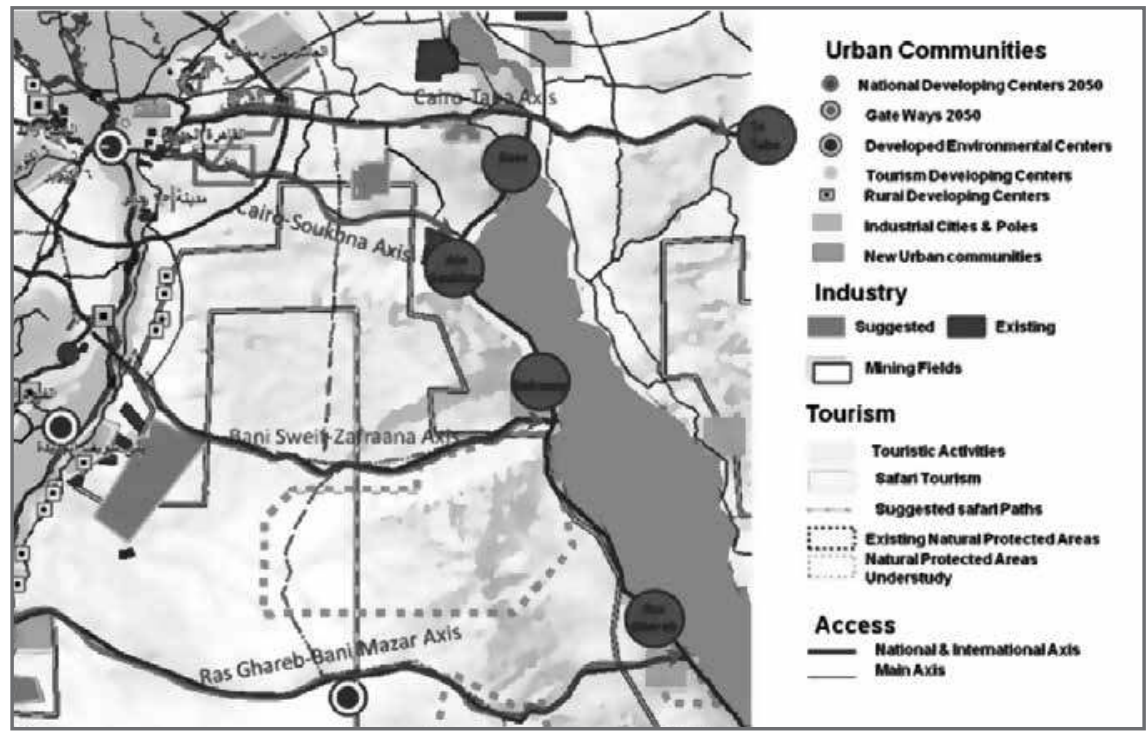

Figure 7 : Le désert oriental et les nouveaux axes de développement, (GOPP, 2011).

\section{VERS UNE PLANIFICATION PARTICIPATIVE ? LES PROPOSITIONS DE LA SOCIÉTÉ CIVILE DEPUIS 2011}

Après la Révolution de 2011, trois organisations non gouvernementales se sont fait remarquer en préparant des contrepropositions à la planification urbaine officielle. Elles ont été à l'initiative de stratégies territoriales importantes qui ont largement circulé au sein de la société civile égyptienne mais aussi auprès de certains membres du gouvernement. Ces projets représentent la réaction d'une société civile longtemps empêchée de participer au processus décisionnel concernant l'amélioration de ses conditions de vie en ville.

L'hôtel Fairmont à Héliopolis fut le théâtre, le 27 Mars 2013, de la signature d'un " pacte civil » entre ces trois ONG. Ce pacte a été signé dans le but de faire pression sur le gouvernement afin qu'il considère leurs propositions. Au cours de cette conférence de presse, les trois organisations, Remal, Egypt Vision 2030 et Development Corridors ont 
fait la promotion de leurs visions et stratégies pour l'aménagement du territoire égyptien. Remal a par exemple présenté son propre schéma directeur, intitulé Egypt 712, en insistant sur le fait que ce dernier peut être amendé à tout moment par la société civile qui peut suggérer des modifications sur la forme comme sur le fond.

Voyons ici quels sont les grands enjeux de ces stratégies postrévolutionnaires, avant de nous interroger sur leurs limites, leur validité et leur efficacité dans le contexte actuel.

\section{Egypt Vision 2030}

"L'avenir appartient au Peuple » s'exclamait la représentante de I'organisation Egypt Vision 2030 lors de la conférence de presse du 27 Mars 2013 à Héliopolis. Contrairement à Egypt 712 et Corridors de Développement, il ne s'agit pas tant d'un plan stratégique que $d^{\prime} u n$ document de prospective généraliste. Cette vision décrit les objectifs souhaités en termes de qualité de vie par un échantillon d'Egyptiens : améliorer le niveau de vie de tous les habitants en assurant la justice sociale et la sécurité ; mettre l'accent sur l'éveil culturel ; adopter les principes du développement durable ; mettre l'accent sur l'importance de la santé et de l'éducation pour le développement humain ; assainir le milieu des affaires en Egypte; promouvoir une économie nationale libérale et compétitive au niveau mondial en suivant les exemples de la Malaisie, la Corée du Sud, le Brésil et la Turquie. Pour ce faire, Egypt Vision 2030 n'hésite pas à faire des projections précises mais idéalisées et pour certaines peu réalistes : augmentation du PIB de $10 \%$ et du revenu moyen par habitant à 12000 dollars par an ; réduction du chômage à $5 \%$; limitation de la croissance démographique annuelle à $1,4 \%$ afin que la population ne dépasse pas le seuil de 105 millions d'habitants en 2030 ; atteindre $100 \%$ d'alphabétisation, etc.

\section{Corridors de développement}

La stratégie "Corridors de Développement » n'est pas née après la révolution de 2011. Elle a été présentée, il y a 20 ans, sous le régime de Moubarak. II s'agit d'un projet d'autoroute jamais réalisé, à l'ouest du Nil, qui relierait la côte Méditerranéenne au lac Nasser (Figure 8). La proposition de Farouk $\mathrm{El} \mathrm{Baz}{ }^{3}$ est aujourd'hui sérieusement réétudiée par la société civile et le secteur privé (investisseurs locaux, arabes et

3. Farouk El-Baz est un scientifique égypto-américain né le 2 janvier 1938 à Zagazig, dans le Delta du Nil. Il a été le directeur du centre pour la télédétection à l'université de Boston. Il a participé au programme Apollo, travaillant notamment sur les programmes de sélection des sites d'atterrissage sur la Lune et sur l'entraînement des astronautes pour l'observation et la photographie lunaires. 
internationaux). Le projet offre une possibilité de développement de nouvelles communautés urbaines, de l'agriculture, de l'industrie, du commerce et du tourisme autour d'un axe de circulation de $2000 \mathrm{~km}$ tracé dans le désert occidental. L'objectif principal est de désengorger l'étroite Vallée du Nil tout en exploitant les terres désertiques à proximité des plus fortes densités de populations du pays. Du point de vue de son promoteur, cette proposition offre une solution innovante aux nombreux problèmes qui se posent aujourd'hui en Egypte. En plus de faciliter la circulation des biens et des personnes dans toute I'Egypte, l'autoroute proposée limiterait l'étalement urbain sur les terres agricoles et ouvrirait une myriade de possibilités pour développer des villes nouvelles à proximité des agglomérations surpeuplées. En outre, le désert étant doté de la lumière du soleil abondante et du vent persistant du nord, les énergies renouvelables constitueraient un nouveau moteur de croissance.

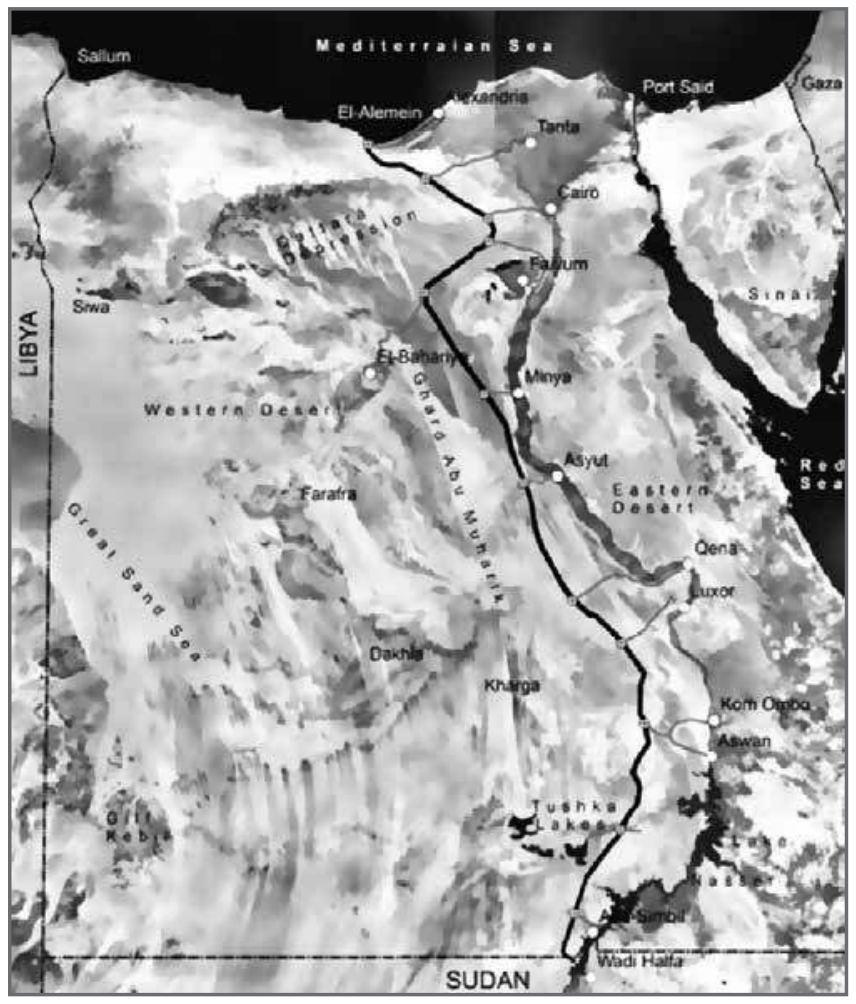

Figure 8 : Les Corridors de Développement, Source : http://faroukelbaz.com 
En plus d'une autoroute construite selon les normes internationales les plus élevées, le projet comprend douze branches Est-Ouest, d'une longueur totale d'environ $800 \mathrm{~km}$, pour relier l'autoroute aux centres de population les plus denses, un chemin de fer pour le transport rapide parallèle à l'autoroute, des canaux acheminant l'eau dessalée, une ligne électrique fournissant de l'énergie nécessaire à la réalisation des premières phases de développement, etc.

L'un des douze axes transversaux permettrait d'accéder au Caire par l'axe Le Caire-Alexandrie, autorisant ainsi l'utilisation du transport terrestre de fret entre Alexandrie et Suez (mer Méditerranée et mer Rouge) comme une alternative au canal de Suez. Cette branche orienterait donc l'extension de l'urbanisation du Caire vers I'ouest, inversant ainsi la tendance actuelle.

\section{Egypte 712}

La stratégie «Egypt 712» ${ }^{4}$ est proposée par I'ONG Remal, en collaboration avec une initiative de la société civile née après la révolution et nommée "Amar Ya Masr» ${ }^{5}$. Cette idée a émergé chez quelques architectes et urbanistes qui ont constitué, avec de jeunes ingénieurs et des professeurs d'universités égyptiennes, un groupe de réflexion entre février 2011 et juillet 2012 qui a animé des conférences et des ateliers de travail, collecté des informations, des articles et des études concernant le développement de l'Egypte.

Basée sur 7 dimensions du développement durable (humaine, spirituelle, sociale, culturelle, économique, politique et écologique), la philosophie principale de cette stratégie est de " revenir vers le futur », en considérant l'Egypte comme un Etat " humain, scientifique, industriel, technologique, culturel, touristique et vert ", ce qu'elle a " toujours été ». Le slogan de ce gigantesque projet est « l'Égypte pour tous les Égyptiens » et sa priorité est de provoquer la "renaissance égyptienne " basée à la fois sur le système tripartite énergie/eau/terre et sur la démocratie et l'équité sociale.

Le résultat de cette vision consiste en un plan stratégique global qui prend en compte les défis, les potentiels et les faits économiques actuels. Il intègre également certaines stratégies proposées par "Corridors de Développement » (Figure 11) et "Egypt Vision 2030 ». Les concepteurs de Egypt 712 proposent de réorganiser les régions égyptiennes en fonction de leurs potentialités économiques. Par conséquent, 12 nouvelles régions, économiquement complémentaires et socialement

4. Le nom « Egypt 712 » réfère à 7 dimensions du développement et à 12 nouvelles « régions » proposées.

5. Amar Ya Masr signifie en arabe que l'Egypte est urbanisée depuis toujours. 
équilibrés seraient créées (Figure 9). En outre, l'Egypte serait dotée de 7 nouvelles « capitales» (Figure 10). A chaque capitale seraient donnés un nom et une fonction basés sur sa position géographique ainsi que sur ses ressources et ses potentiels. Outre Le Caire qui serait la capitale " culturelle et spirituelle », il y aurait Néfertari (capitale administrative et de loisirs) ; Alexandrie (capitale de la science et de connaissance) ; Sennine (capitale de la finance, du commerce et des affaires); Louxor (capitale du patrimoine); Al-Waha ou l'Oasis (capitale de la technologie verte); Toshka (un nouveau centre pour l'agriculture biologique). Concernant Le Caire, plusieurs projets de développement intégrés sont envisagés, comme pour le quartier populaire Mansheyat Nasser. Une équipe de jeunes architectes a déjà participé avec les habitants du quartier à la réhabilitation de plusieurs bâtiments et au nettoyage de quelques rues.

Sur ce même modèle de spécialisation fonctionnelle, l'aménagement de 12 nouvelles villes est également envisagé : la ville de Néfertari située à l'ouest de Saloum, spécialisée dans les sciences astronomiques, la ville de Sinaï, au sud de Rafah, dans l'énergie nucléaire, le Nouveau Delta dans I'agriculture moderne, etc. De même, le plan d'aménagement de 12 villes existantes sera entièrement repensé : Suez, Banha, Damiette, Siwa, Mansoura, etc.

Un certain nombre de critiques ont été faites à ce qui pouvait apparaître comme un zoning administratif et fonctionnel favorisant la partition de l'Egypte. En outre, plusieurs urbanistes reprochent à Egypt 712 son caractère utopique et le manque de rigueur qui caractérise ses différentes propositions. Au cours des différentes réunions publiques qu'elle a organisé, I'ONG Remal a rappelé que rien n'était figé et que la société civile pouvait encore faire évoluer les propositions et organiser d'autres ateliers de travail sur le sujet. Remal a enfin proposé que le gouvernement actuel nomme 50 spécialistes capables d'élaborer une sorte de " constitution » du développement urbain en Egypte, soumise au peuple par referendum. 


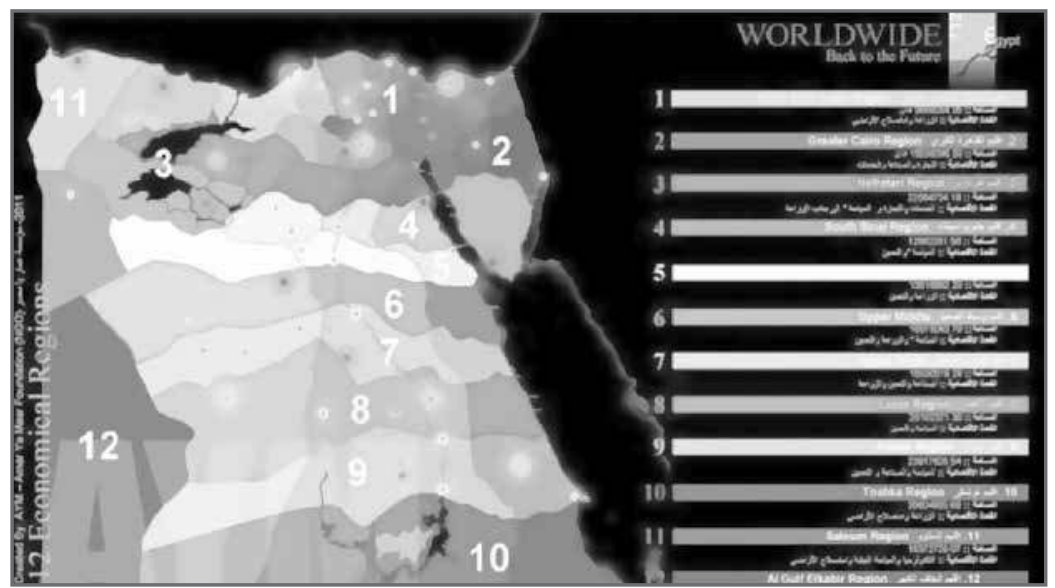

Figure 9 : 12 Régions Economiques, Egypt 712, (Remal, 2011).

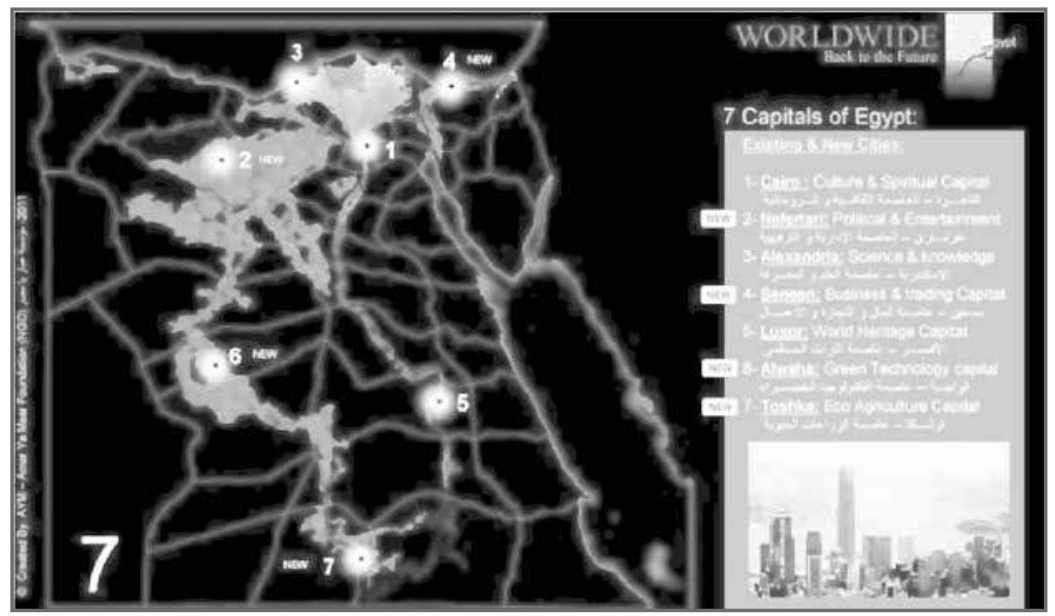

Figure 10 : Les 7 nouvelles capitales égyptiennes. (Egypt 712), 2011.

\section{CONCLUSION}

Décentraliser le Caire est aujourd'hui largement considéré comme un objectif relativement utopique. Beaucoup d'égyptiens admettront en outre volontiers que s'éloigner du Caire est aussi compliqué que de continuer à y vivre. Les autorités responsables de la planification stratégique de la capitale égyptienne font face à de nombreux problèmes, dont celui de convaincre les habitants du Caire que la vie est possible en dehors. Il faut aussi admettre le fait que la planification gouvernementale envisagée pour le Grand Caire depuis les années soixante n'a pas permis de résoudre les grands problèmes de la capitale égyptienne. Au contraire, chaque nouveau plan stratégique élaboré 


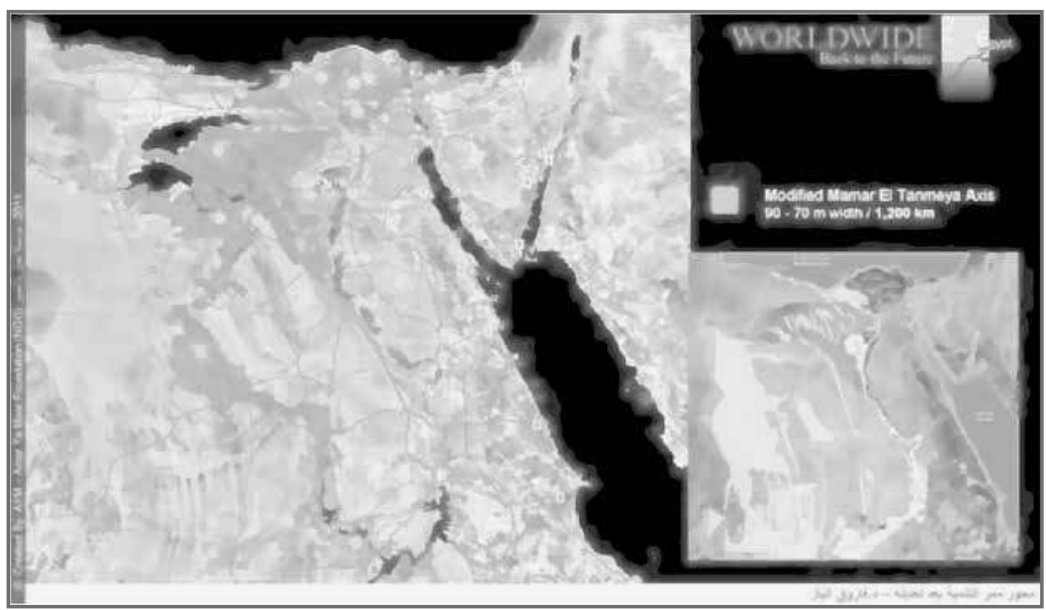

Figure 11 : La Modification des Corridors de Développement, Egypt 712, (Remal, 2011).

depuis les années 1970 s'est accompagné de nouvelles complications urbaines inattendues et toujours très préoccupantes aujourd'hui (zone d'urbanisation informelles, grignotage de la terre agricole, congestion de la capitale, etc.).

En outre, d'un côté le rôle du Caire et le futur de son développement urbain ne semblent pas assez bien définis par les documents de planification stratégique officiels les plus récents. Si le GOPP cherche à démontrer que «Egypt 2052 » est bien une stratégie à la hauteur des exigences formulées par les acteurs de la révolution de 2011, il n'en demeure pas moins que l'institution n'a pas développé de nouvelles méthodes ou techniques "révolutionnaires » pour se faire. Le projet "Egypt 2052 » lui-même a été conçu par les mêmes experts, dans les mêmes conditions et avec les mêmes données que son prédécesseur Cairo 2050.

D'un autre côté, un vent de reforme urbaine souffle sur la société civile aujourd'hui. On assiste ainsi à l'apparition ou à la réhabilitation de plusieurs stratégies de planification urbaine plus ou moins novatrices, plus ou moins utopiques. Ces projets incarnent la contestation de la politique aménagiste de l'ancien régime mais aussi des politiques portées par les gouvernements de transition qui se sont succédé depuis 2011. Une série de consensus s'installe au sein de la société civile concernée par ces questions : d'une part il faut absolument désengorger la capitale tout en lui réservant la place qu'elle mérite dans une politique d'aménagement nationale. D'autre part, les sociétés civiles impliquées dans ce débat se montrent intransigeantes en matière de justice sociale, d'économie stable, de qualité de vie, d'administration locale et de participation des habitants aux processus décisionnels. 
RANDA A. MAHMOUD, Architecte, Urbaniste est née en 1974 en Egypte. Après avoir fini ses études doctorales en France à I'IUP, elle est aujourd'hui Professeur Adjoint au Département de Planification Urbaine et de Conception à l'Université Ain Shams et également au Département d'Architecture à l'UFE (Université Française d'Egypte) où elle enseigne Conception Architecturale, Conception Urbaine, Paysage Urbain et Histoire de la Cité.

Elle a été impliquée, en tant que Freelancer dans de nombreux projets résidentiels et de conception urbaine. Elle a été nommée au prix de I'Agha Khan Award for Architecture- cycle 2013 pour le projet «Beit Âl Rawi », une villa résidentielle au Nouveau Caire. Elle a plusieurs publications locales et internationales dans les domaines de morphologie urbaine, typologie architecturale, morphologie sociale, quartiers d'urbanisation spontanée, aménagement de déchets ménagers et l'espace urbain cairote post révolutionnaire.

AHMED S. ABD ELRAHMAN est Professeur Adjoint au Département de Planification Urbaine et de Conception à I'Université Ain Shams. II possède une vaste expérience dans le domaine du développement durable en mettant l'accent sur des projets à grande échelle d'habitation, le développement du front de mer, le développement local, et l'aménagement du littoral et la participation du public.

Il a été impliqué dans l'enseignement de Conception Architecturale, Urbanisme, Conception Urbaine et l'aménagement paysager, ainsi qu'au développement du secteur riverain pour les étudiants en master à I'Université Ain Shams. Il a également enseigné d'enseigner aux autres Ecoles d'Architecture comme : (AAST) Académie arabe des sciences et de la technologie, (MIU) Université internationale de l'Egypte, (BUE) I'Université Britannique en Egypte.

Il a été également impliqué dans de nombreux projets de conception urbaine et d'urbanisme des où il a une telle expérience pendant son travail dans PUD ( Planification et Développement Urbain ) Cabinet de conseil, qui a conçu des projets de méga planification et d'aménagement urbain en Egypte et de nombreux pays du Moyen-Orient, en plus de son expérience pratique grâce à son travail avec les organismes nationaux et internationaux comme NOUH, PPPCO, ONU-HABITAT.

Il a plusieurs publications locales et internationales sous les titres de " développement du secteur riverain », " taudis », «friches industrielles», « espaces urbains après les révolutions ». 
RANDA A. MAHMOUD, Architect, Urban Planner was born in 1974 in Egypt. After having finished her doctoral studies in France at the IUP, she is now an Assistant Professor at the Department of Urban Planning and Design in Ain Shams University and also at the Department of Architecture at the UFE (French University of Egypt) where she teaches Architectural Design, Urban Design, Urban Landscape and History of the City.

She has been involved as a Freelancer in many residential and urban design projects. She was nominated for the price of the "Aga Khan Award for Architecture-cycle 2013" for the project "Beit Âl Rawi, » a residential villa in New Cairo. She has several local and international publications in the fields of urban morphology, architectural typology, social morphology, informal urbanization, households' waste management and Cairo's post-revolutionary urban space.

AHMED S. ABD ELRAHMAN is an Assistant Professor at Urban Design \& Planning department - Faculty of Engineering - Ain Shams University; he has extensive experience in sustainable development with particular emphasis on large scale residential projects, waterfront development, local development, coastal planning and public participation.

He has been involved in teaching Architecture Design, Urban Planning, Urban Design and landscape design for undergraduates. And Waterfront development for the post graduates students in Faculty of Engineering - Ain Shams University. In addition to teaching experiences at other colleges like (AAST) Arab academy for science \& technology, (MIU) Misr International University, (BUE) the British University in Egypt.

He was involved in many urban design \& urban planning projects where he has such experience during his work in PUD (Planning and Urban Development) Consulting firm, which has been implementing mega planning \& urban design projects in Egypt and many countries in the middle East, in addition to his practical experience through his work with national and international entities like NOUH, GOPP, UN-HABITAT.

He has several local \& international publications under titles of «waterfront development», «Slums», «brownfields», «urban spaces after revolutions». 


\section{REFERENCES}

Barthel P.-A., "Repenser les « villes nouvelles » du Caire : défis pour mettre fin à un développement non durable », Égypte/Monde arabe, Troisième série, Développement durable au Caire : une provocation?, [En ligne], mis en ligne le 01 septembre 2012. URL: http://ema.revues.org/2990

Barthel P.-A., Mongid (S.), Le Caire, réinventer la ville, Collection «Villes en mouvement», $n^{\circ}$ 21, Editions Autrement, 2011.

DenIS É., «Etirement Cair ote et mobilités résidentielles/pendulaires», in : Mégalopoles transports et mobilités : Confrontations, 9 Avril 2008.

Denis E., Vignal L., "Dimensions nouvelles de la métropolisation dans le Monde arabe: le cas du Caire », dans "Cahiers de la Méditerranée», Vol. 64 : Les enjeux de la métropolisation en Méditerranée, http://cdlm.revues.org/75, 2005.

EL KADI G., «La régénération du centre-ville du Caire : le pari de la durabilité», Égypte/Monde arabe, Troisième série, Développement durable au Caire : une provocation ?, [En ligne], 2011. URL: http://ema.revues.org/2995.

Elnaggar A., Bedier M., Mubarak R., "Urban and peri-urban agriculture: towards better understanding of low income producers' organizations", UPA-Cairo Case study Final report-FAO, IDRC, MALR-AERI, Egypt, Cairo Case Study Report, Agricultural Economic Research Institute, Cairo, Egypt, October 2006.

General Organization for Physical Planning (GOPP), «Vision of Cairo 2050 within a national vision of Egypt», Cairo, 2010. — «Vision of Egypt 2052», Cairo, 2011.

GTZ, "Cairo's informal areas between urban challenges and hidden potentials, Facts. Voices. Visions», le Caire, 2009, 224 P.

KIPPER (R.), "Cairo: A Broader View», in "Cairo's informal areas: Between urban challenges and hidden potentials", Edition R. KIPPER \& M. FISCHER, 13-16. Portugal: Norprint SA, 2009.

Madbouly M., The Strategic Urban Development Plan for Greater Cairo Region "Cairo 2050", General Organization for Physical Planning (GOPP), Cairo, Egypt, 2010.

Ministry of Housing, Utilities \& Urban Development (MOHUUD), Japan International Cooperation Agency (JICA), General Organization of Physical Planning (GOPP), (2008), "The Strategic urban development master plan study for sustainable development of The Greater Cairo Region in The Arab Republic of Egypt, final report», Vol. 1: Summary, August 2008.

Piffero E., "Beyond Rules and Regulations: The Growth of Informal Cairo", in Cairo's informal areas: Between urban challenges and hidden potentials, Edition R. KIPPER \& M. FISCHER, 21-28. Portugal: Norprint SA, 2009.

- What happened to participation? Urban development and authoritarian upgrading in Cairo's informal neighborhoods, Nabu Press, 2012, 224 P.

SIMS D., Development Benefits of the Cairo Ring Road and associated Bridges, Rapport final soumis au USAID, Le Caire, Egypte, 20 Août 1990.

— "Understanding slums: Case studies for the global report on human settlements. Urban slums report: the case of Cairo, Egypt", in: UNHabitat Global Report on Human Settlements 2003, The Challenge of Slums, Earthscan, London; Part IV: 'Summary of City Case Studies', 2003, pp. 195-228. 
United Nations Human Settlements Program (UN-HABITAT), "Cairo: A city in transition", in "Cities \& citizens series, bridging the urban divide», Vol. 2, 2011, 177 p.

TARBush N., "Cairo 2050: Urban Dream or Modernist Delusion? », in "Journal of International Affairs", Vol. 65, n² 2, Spring/Summer 2012.

World Bank, Sustainable Development Department, Middle East and North Africa Region, Arab Republic of Egypt: Urban Sector Update, Vol. 1, 2008. 